\title{
MEDIDAS ELETRENCEFALOGRÁFICAS DURANTE A APRENDIZAGEM DE TAREFA MOTORA SOB EFEITO DO BROMAZEPAM
}

\author{
Victor Hugo Bastos', Dionis Machado², Marlo Cunha33, Claudio Elidio Portella4, \\ Maurício Cagy, Vernon Furtado ${ }^{6}$, Roberto Piedade7, Pedro Ribeiro ${ }^{8}$
}

\begin{abstract}
RESUMO - Neuromoduladores alteram constantemente as relações neurais pré-existentes no sistema nervoso. O bromazepam é utilizado com freqüência na prática clínica para diminuir padrões de ansiedade. Poucos são os experimentos correlacionando este ansiolítico às tarefas motoras. Neste contexto, o presente experimento visa analisar as alterações motoras e eletro c o rticais decorrentes da administração de diferentes doses de bromazepam mediante a prática motora, e relacionar o efeito da droga a performance motora mão-dominante versus não dominante. Sujeitos saudáveis (39), de ambos os sexos, entre 20 a 30 anos compuseram a amostra. Os grupos controle (placebo) e experimental (bromazepam de $3 \mathrm{mg}$ e $6 \mathrm{mg}$ ) foram t reinados na tarefa de datilografia num modelo duplo-cego randomizado. Resultados do teste Stroop (atenção) não demonstraram diferenças no escore bruto e no tempo de execução do mesmo. Em contrapartida, nos resultados comportamentais foram observados um efeito principal entre blocos nas variáveis tempo de execução e erros cometidos durante a pratica motora. Os dados eletrofisiológicos evidenciaram interações significantes para: lateralidade/condição/momento; lateralidade/condição; lateralidade/momento; condição/momento; condição/setor.
\end{abstract}

PALAVRAS-CHAVE: bromazepam, EEGq, aprendizagem motora, controle motor.

\begin{abstract}
Electroencephalography measures in motor skill learning and effects of bromazepam
ABSTRACT - Neuromodulators change brain's neural circuitry. Bromazepam is often been used in the pharmacological treatment of anxiety disorders. Few papers links this anxiolytic to motor tasks. The purpose of this study was to examine motor and electrophysiological changes produced by administration of bromazepam in differents doses $(3 \mathrm{and} 6 \mathrm{mg}$ ). The sample consisted of 39 healthy individuals, of both sexes, between 20 and 30 years of age. The control (placebo) and experimental (bromazepam $3 \mathrm{mg}$ and bromazepam $6 \mathrm{mg}$ ) groups were submitted to a typewriting task, in a randomized, double-blind design. The results did not reveal differences on score and time of the attention test. In the comportamental analysis was noticed blocks as main effect to behavioral variables (time and mistakes in the task). Electrophysiological data showed significants interactions to: laterally/condition/moment; laterally/condition; laterally/moment; condition/moment; condition/site.
\end{abstract}

KEY WORDS: bromazepam, qEEG, motor learning, motor control.

Durante a vida nos deparamos com diversos fatores tais como agitação, pressão e stress. O somatório destes fatores pode levar ao sentimento de sofrimento que representa a tentativa humana de viver num ambiente que lhe parece adverso. A ansiedade é uma das manifestações mais freqüentes do sofrimento e pode ser descrita como um estado particular de esperar ou pre parar-se para o perigo, ainda que desconhecido. Atualmente a ansiedade vem se configurando como problema de saúde pública, visto os prejuízos causados por esta na qualidade de vida do indivíduo ${ }^{1,2}$. Inúmeras terapias vêm sendo empregadas no sentido de proporcionar melhor qualidade de vida aos porta do res desta doença. Benzodiazepínicos têm sido amplamente utilizados nas últimas quatro décadas para o tratamento dos transtornos de ansiedade. Tal uso justifica-se por suas propriedades ansiolítica, anticonvulsiva, miorelaxante e hipnótica em caso de dose elevada ${ }^{3-7}$. Compostos benzodiazepínicos

\footnotetext{
'Doutorando, Laboratório de Mapeamento Cerebral e Integração Sensório-Motora - Instituto de Psiquiatria (IPUB) - Universidade Federal do Rio de Janeiro Rio de Janeiro RJ Brasil (UFRJ); ${ }^{2}$ Mestranda (IPUB/UFRJ); ${ }^{3}$ Mestrando (IPUB/UFRJ); ${ }^{4}$ Iniciação Científica UNIABEUFisioterapia (IPUB/UFRJ) ${ }^{5} \mathrm{D}$. Sc. Em Engenharia Biomédica, COPPE - IPUB (UFRJ); ${ }^{6} \mathrm{P}$ rofessor Titular, PROCIHM ${ }^{7} \mathrm{P}$ rofessor Adjunto III Doutor, (IPUB/UFRJ); ${ }^{8}$ P rofessor Adjunto II PhD EEFD - IPUB (UFRJ); Professor Pesquisador, Universidade Castelo Branco (PROCIHM).
} 
atuam como neuromoduladores, aumentando a afinidade dos receptores de ácido gama aminobutírico (GABA) pelo seu neurotransmissor ${ }^{8,9}$. Estudos comprovam a eficácia da propriedade ansiolítica dos benzodiazepínicos nos transtornos de ansiedade, em contrapartida alguns estudos demonstram efeitos deletérios desta droga em tarefas que envolvam integração-sensório-motora, dentre os quais podem ser destacados: certo grau de incoo rdenação motora, aumento do tempo de reação, perturbação da marcha e déficit de memória imediata $^{4,10-12}$.

Tarefas motoras fazem parte da rotina diária do ser humano. São freqüentes os momentos em que um indivíduo busca um movimento, ou mesmo planeja um gesto motor. Particularmente questões motoras envolvendo aprendizagem têm sido investigadas através de várias metodologias ${ }^{13}$. Medidas eletrencefalográficas EEG vêm sendo utilizadas a fim de se analisar processos motores relacionados à aprendizagem ${ }^{14}$, bem como para avaliar alterações eletrocorticais decorrentes da administração de drogas com efeito sobre o sistema nervoso central (SNC) ${ }^{15}$.

Este estudo tem por objetivo: (1) analisar as alterações motoras e eletrocorticais decorrentes da administração de diferentes doses de benzodiazepínico (bromazepam) mediante a prática de tare fa motora; e (2) relacionar o efeito da droga a performance motora mão-dominante versus não dominante.

\section{MÉTODO}

Amostra - Foram selecionados 39 estudantes de graduação não portadores de comprometimento da saúde física e/ou mental (anamnese prévia). Excluíramse os usuários de substâncias psicotrópicas ou psicoativas; indivíduos que tiveram sono inferior ao período de 6 a 8 horas na noite anterior ao experimento; experientes em datilografia, dominância manual sinistra de acordo com o inventário de Edinburgh ${ }^{16}$. Todos os sujeitos tinham se alimentado normalmente até duas a três horas antes do experimento. Os participantes foram notificados verbalmente do modelo experimental em linguagem clara e autorizaram sua participação no modelo de estudo assinando termo de livre consentimento (no qual estava descrito o resumo do modelo experimental). 0 experimento foi aprovado pela Comissão de Ética do Instituto de Psiquiatria da Universidade Federal do Rio de Janeiro.

Desenho experimental-Em um primeiro momento, ao chegar no laboratório, o participante era informa do sobre os detalhes do estudo e suas possíveis repercussões.
Após concordar e assinar a declaração de consentimento tinha início a primeira captação de sinal por meio do EEG. Esta primeira captação consistia em seis minutos nos quais o sujeito mantinha-se de olhos fechados e seis minutos de olhos abertos, sendo solicitado evitar piscadas a fim de minimizar a quantidade de artefatos musculares no EEG. Em seguida o sujeito ingeria uma cápsula que poderia conter placebo (amido $400 \mathrm{mg}$ ) ou $3 \mathrm{mg}$ de bromazepam ou $6 \mathrm{mg}$ de bromazepam. A distribuição das cápsulas seguiu um modelo duplo-cego randomizado. Após a ingesta o sujeito mantinha-se sentado na cadeira de exame até o momento do treinamento motor. Trinta minutos após a ingestão da cápsula era feita uma avaliação do nível de atenção do sujeito pelo teste de Stroop. Após o teste era iniciada nova captação, desta vez simultaneamente à tarefa da datilografia. Ao término da tarefa era realizada mais uma etapa de captação (seis minutos olhos fechados e seis minutos olhos abertos).

Aquisição de dados de EEG - O sinal de EEG foi captado utilizando o aparelho Braintech 3000 (EMSA - Inst rumentos Médicos, Brazil), o qual apresenta uma placa conversora analógica-digital (A/D) de 32 canais com resolução de 12 bits, colocada em um slote ISA de um Pentium III - com um processador de $750 \mathrm{~Hz}$. Os eletrodos encontravam-se dispostos em uma touca de acordo com o sistema internacional 10-2017, incluindo-se os eletrodos de referência posicionados nos lóbulos das orelhas (biauricular) ${ }^{18}$. A seleção do tamanho da touca era feita mediante ao perímetro cefálico de cada sujeito (toucas de tamanhos diferenciados). A captação do sinal do EEG foi realizada em sala preparada com isolamento acústico e elétrico. A iluminação da sala foi reduzida durante a aquisição do sinal, exceto no momento da tarefa.

A diferença entre o potencial elétrico de um determinado eletrodo (prata niquelada) no escalpo e a referência auricular resultou no sinal adquirido do eletrodo em questão. Os níveis de impedância de cada eletrodo foram verificados e fixados entre $5-10 \mathrm{~K}$ ohms $(\theta)$ e mantidos nesses padrões. Os sinais adquiridos deviam estar com o total de amplitude (pico a pico) menor que 100 $\mu \mathrm{V}$. Desta forma, o sinal deveria ser amplificado com ganhos variando de 20000 a 50000 . Os sinais de EEG adquiridos deveriam flutuar entre $0,01 \mathrm{e} 50 \mathrm{~Hz}$. Dois eletrodos de $9 \mathrm{~mm}$ de diâmetro montados de forma bipolar pe rmitiram estimar a atividade elétrica ocular. Os eletrodos foram posicionados, respectivamente, acima e abaixo da órbita do olho direito para registrar movimentos oculares verticais e no canto externo (canthus) do mesmo para registrar movimentos oculares horizontais.

Os parâmetros do EEG quantitativo foram extraídos de trechos de 2 segundos antes e depois do início do movimento em 20 derivações de acordo com o sistema 1020 de posicionamento de eletrodos. Para garantir uma seleção mais criteriosa foram retirados os trechos contaminados por artefatos musculares através de algoritmo de rejeição automática por trespasse de limiar (100 $\mu \mathrm{V})$ 
e por inspeção visual. A partir da estimativa da densidade espectral de potência, obteve-se a potência nas bandas do EEG (delta, teta, alfa e beta) antes do início do movimento (entre -2 s e $0 \mathrm{~s}$, considerado como referência - R) e após o movimento (entre 0 s e 2 s, denominado P), com relação a cada pressionamento de tecla de interesse. Em 0 s tem-se a representação do momento exato do pressionamento da tecla de interesse. Além da potência em cada banda, a potência total também foi estimada visando à obtenção da potência relativa, definida como a razão entre a potência em cada banda e a potência total. Com o objetivo de facilitar a visualização, todos trechos de sinal contidos entre 2 s antese 2 s após o pressionamento da tecla de interesse sofreram filtragem passa-faixa (associação de filtros passa-altas e passabaixas Butterworth de ordem 2 cada). Uma vez filtrados os respectivos trechos de dados, estes foram elevados ao quadrado e sofreram filtragem passa-baixas objetivando a detecção de envoltória. Uma promediação de todas as trilhas ocorreu para se obter à evolução temporal da potência média em todas as 20 derivações utilizadas, que pode ser interpretado visualmente e servir de suporte às conclusões estatísticas.

Procedimento da tarefa motora - A máquina de datilografia selecionada para este trabalho foi uma Olivetti modelo Línea 98, estilo antigo. Tal opção se deve ao fato da representação cortical ser maior quanto maior o grau de força empregada na tarefa ${ }^{19,20}$. Neste tipo de máquina de datilografia a exigência de força por parte dos músculos intrínsecos e extrínsecos da mão é maior do que quando comparada à força exigida por um teclado de computador (hipótese confirmada por estudos pilotos e avaliações prévias) ${ }^{21-23}$. Os sujeitos estavam sentados de maneira confortável em cadeira com suporte para os antebraços a fim de diminuir os artefatos musculares. A distância entre a cadeira do sujeito e a mesa onde se encontrava a máquina de datilografia foi de cerca de $20 \mathrm{~cm}$ variando em função do comprimento do antebraço de cada sujeito. O teclado da máquina foi coberto com uma caixa de madeira que evitava a visualização do posicionamento das mãos sobre o teclado e que exigia do sujeito a criação de uma "referência espacial" para o teclado.

A tarefa consistiu num método de datilografia ${ }^{24}$ e era realizada num único dia. O exercício era composto por quatro blocos sendo cada bloco constituído por doze linhas. Cada linha deveria conter cinco seqüências de letras para cada mão. A seqüência de letras para cada mão foi assim estabelecida: asdfg para mão esquerda e çlkjh para mão direita. A seqüência da mão esquerda era iniciada pelo dedo mínimo pressionando a tecla referente à letra a, em seguida ocorria o pressionamento da tecla $s$ pelo anular, tecla $d$ pelo dedo médio, tecla $f$ e $g$ pelo indicador. Terminada a seqüência da mão esquerda era efetuado o pressionamento da tecla espaço, utilizando para isto o polegar esquerdo ou o direito, e então era iniciada a seqüência da mão direita (dedo mínimo pressionando ç, anular na tecla $l$, médio para $k$, indicador para as teclas $j$ e $h$ ).

Abaixo do teclado da máquina de datilografia existia um circuito elétrico responsável pela captação do pressionamento das teclas de interesse, a saber, a e $g$, pois respectivamente representavam início e término da seqüência da mão esquerda, ç e $h$ pelo mesmo motivo em função da mão direita e a tecla espaço que representava mudança para seqüência seguinte. Tal circuito era composto por cinco dispositivos em forma de "U" denominados optoeletrônicos. Cada optoeletrônico possui uma porção emissora e uma porção receptora de luz que permitia a transferência da informação de pressionamento das teclas de interesse para o sistema de aquisição de dados onde eram efetivamente reconhecidas as letras conforme seus traçados gráficos característicos (mediante passagem por um simples conversor digitalanalógico).

Seleção das bandas de freqüência, áreas corticais de in te resse e seleção das teclas de interesse - Neste estudo selecionou-se como teclas de interesse a tecla ç e a tecla a. Esta opção veio do fato destas teclas exigirem demanda cortical motora do hemisfério não dominante do sujeito, direito (tecla a) e do hemisfério dominante, esquerdo (tecla ç). Particula mente neste modelo experimental tais teclas trabalham com o mesmo dedo, só mudando, obviamente, o lado corporal e conseqüentemente o hemisfério ativo nesta tarefa. Desta forma são possíveis comparações entre os dois hemisférios e como eles trabalham em função desta tarefa motora.

A banda de freqüência de interesse neste estudo é a teta. Esta opção justifica-se pelo fato desta estar relacionada a mecanismos de potencialização de longa duração (LTP) e depressão de longa duração (LTD). Tais situações são conhecidas bioquimicamante por relacionarem-se com os mecanismos neurais da aprendizagem. Modificações nos padrões corticais (produzidas experimentalmente) que suprimem as oscilações de teta causam prejuízo no aprendizado, ao passo que drogas conhecidas por aumentar as funções de memória em ratos evidenciam aumento na amplitude teta. Amplitude, freqüência e fase da banda teta mostram-se críticas para o processo de aprendizagem ${ }^{18,25}$. Tem-se a banda teta como eminentemente relacionada aos mecanismos neurais que gerenciam e regulam processos de aprendizagem motora.

Os eletrodos de interesse estão localizados em áreas frontais e parietais. Áreas frontais foram selecionadas em virtude da relação destas aos mecanismos de motivação ${ }^{26}$, planejamento e execução de movimentos voluntários. A inclusão de eletrodos dos lobos parietais justifica-se pelo controle dos mecanismos sensoriais e espaciais que ocorrem em tais áreas ${ }^{8,27}$. Esta seleção tem como objetivo comparar os achados eletrofisiológicos en- 
t re os dois hemisférios cerebrais. Desta forma, foram selecionados os seguintes eletrodos fronto-parietais: FP1, FP2, F3, F4, F7, F8, C3, C4, P3, P4.

Análise estatística - Em relação ao teste de Stroop foi realizada uma ANOVA (one-way) com um Post hoc tipo Scheffe para tempo e escore bruto. Em especial, o Stroop foi realizado 30 minutos após a ingestão da cápsula de placebo ou bromazepam de 3 ou 6 mg, dependendo da randomização. A realização de uma única testagem (Stroop) está em função do efeito aprendizado do teste. Desta forma fica claro o motivo da não realização de uma testagem prévia à ingestão da droga. Particularmente, este teste tem sido utilizado para análise dos processos de atenção em modelos com EEG ${ }^{28}$.

Para análise comportamental foi realizada uma ANOVA (two-way) com um Post hoc tipo Scheffe. Foi considerado, dentro da análise comportamental, o número de erros cometidos por cada sujeito dentro de cada bloco separadamente e o tempo total de execução de cada bloco independentemente. Os erros foram considerados conforme critérios pré-estabelecidos e conferidos pelo autor principal. Este modelo de ANOVA foi utilizado para comparar dois fatores: condição (placebo, bromazepam $3 \mathrm{mg}$ e $6 \mathrm{mg}$ ) e bloco (bloco I e bloco III). Estes blocos foram selecionados, visto que o primeiro indica o momento de inexperiência do indivíduo e o terceiro indica um momento do aprendizado. O segundo bloco não foi selecionado já que o tempo era curto para um aprendizado tão efetivo como provavelmente ocorreria no terceiro bloco. O quarto bloco também não foi selecionado neste modelo, já que parece ser o bloco em que o sujeito apresenta um certo grau de fadiga em relação à tarefa. Foi realizada ainda uma ANOVA (two-way) com um Post hoc tipo Scheffe para correlacionar a lateralidade (mão esquerda versus mão direita) com o número de erros. Desta forma, foram selecionados apenas os erros cometidos no terce i robloco, bloco este que parece estar mais relacionado ao aprendizado como já descrito. Assim, foram comparadas as condições (placebo, bromazepam de $3 \mathrm{mg}$ e de $6 \mathrm{mg}$ ) com o número de erros no terceiro bloco em cada mão.

A análise dos dados eletrofisiológicos considerou a tarefa completa, como um todo, e não a subdivisão em blocos como considerada na análise comportamental. Os valores de potência relativa obtidos (dados eletrofisiológicos) dois segundos antes do pressionamento das teclas a e ç, e dois segundos após o pressionamento, foram transformados para logaritmo em base dez (Log10) utilizando o software SPSS versão 10.0. Esta transform ação se fez necessária porque os valores de potência relativa não apresentam distribuição Gaussiana. Testes de homocedasticidade demonstraram que os dados em Log10 apresentam distribuição normal para estes dados eletrofisiológicos ${ }^{29}$. Em relação aos dados eletrofisiológicos, uma ANOVA (four-way) comparou os fatores: lateralidade (mão esquerda em relação à tecla a e mão direita em relação à tecla ç); condição (placebo x bromazepam 3mg x bromazepam 6mg); momento (dois segundos antes pressionamento das teclas a e ç, e dois segundos após do pressionamento da tecla) e setores no escalpo (hemisfério esquerdo - FP1, F3, C3, F7, P3 e direito - FP2, F4, C4, F8, P4). Utilizou-se um teste Post hoc tipo Scheffe. Formou-se um modelo estatístico com medidas repetidas dentro dos fatores onde se tem: condição (3); lateralidade (2); momento (2); setor (2). Para todas as análises foi considerado um p estatístico menor ou igual a 0,05 ( $\mathrm{p} \leq 0,050)$.

\section{RESULTADOS}

Os resultados obtidos foram divididos em três categorias conforme as variáveis dependentes. Desta maneira, foi montado um modelo com dados de três variáveis, sendo: as neuropsicológicas, comportamentais e eletrofisiológicas.

Variável neuropsicológica (nível de atenção) A análise da atenção (teste de Stroop) não mostrou interação entre os grupos placebo e experimental, nem em relação a variável tempo $(p=0,985)$ nem para o escore bruto dos dados $(p=0,363)$.

Variáveis comportamentais - Os resultados da ANOVA (two-way), condições (placebo e experimental) versus blocos (bloco I e bloco III), na variável tempo de execução da tarefa (Fig 1) apresentou um efeito principal para blocos $(p=0,000)$. Em especial, não houve interação entre condição versus blocos, e não foi demonstrado efeito principal entreas condições. A ANOVA (two-way), condições

\section{Condição $\mathrm{x}$ blocos $\mathrm{x}$ te mpo}

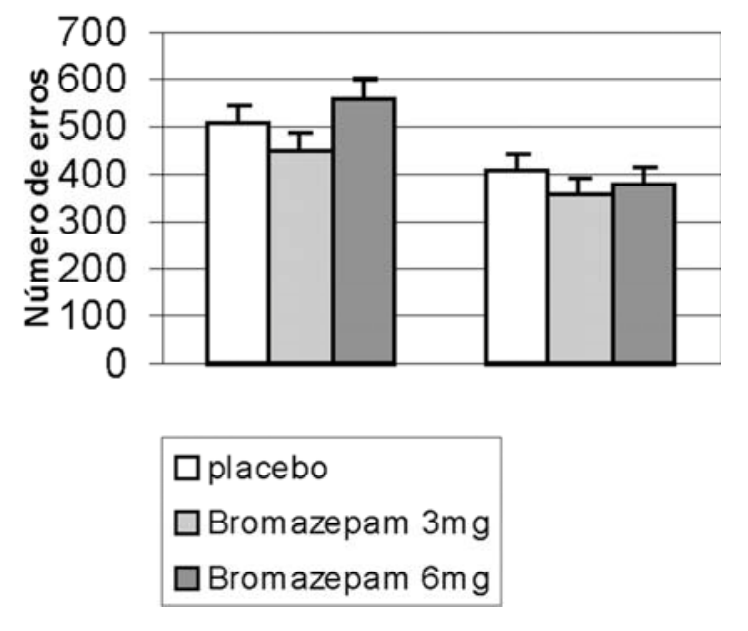

Fig 1. Relação entre o tempo de execução dos blocos I e III nas diferentes condições. 


\section{Condição $\mathrm{x}$ blocos $\mathrm{x}$ erros}

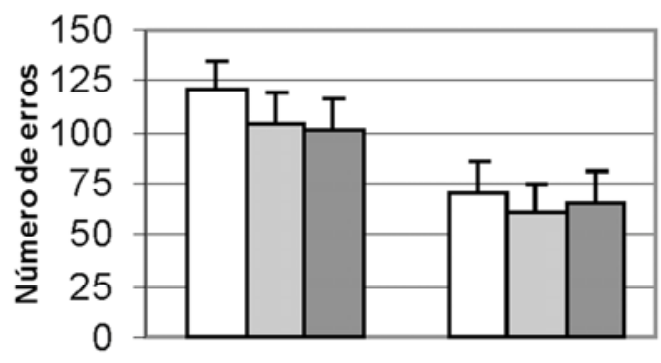

$$
\begin{array}{|l}
\square \text { placebo } \\
\square \text { Bromazepam 3mg } \\
\square \text { Bromazepam } 6 \mathrm{mg}
\end{array}
$$

Fig 2. Relação entre o número de erros nos blocos I e III nas diferentes condições.

e blocos, no número de erros (Fig 2), apresentou efeito principal entre blocos $(p=0,001)$. Não foram observadas interações entre condição versus blocos, e não houve diferença significativa entre as condições. Na comparação realizada entre os erros do terceiro bloco e o fator lateralidade foi observado efeito principal para lateralidade $(p=0,001)$ sem evidências de interações entre erros no terceiro bloco e lateralidade.

Variáveis eletrofisiológicas - Em relação aos dados eletrofisiológicos os valores considerados foram os da potência relativa na banda teta dois segundos antes do pressionamento da tecla a e dois segundos depois do pressionamento (momentos em relação à tecla a) e suas possíveis interações com dois segundos antes do pressionamento da tecla ç e dois segundos depois do pressionamento (momentos em relação à tecla ç). Os achados da ANOVA fourway mostraram significância em diversos fatores isoladamente (vários efeitos principais) e em diversas interações (interações entre os fatores estudados). Particularmente todos os quatro fatores da ANOVA analisados (condição, momento, lateralidade, setor) foram, significantes, evidenciando-se que todos funcionam como efeitos principais. Desta forma foram encontrados os seguintes valores para os graus de liberdade, $\mathrm{F}$ estatístico e níveis de significância: lateralidade $\left(F_{(3,1556)}=92,541 \mathrm{p}=0,000\right)$; condição $\left(\mathrm{F}_{(3,1556)}=6,171 \mathrm{p}=0,002\right)$; momento $\left(\mathrm{F}_{(3,1556)}\right.$ $=436,189 \mathrm{p}=0,000)$; setor $\left(\mathrm{F}_{(3,1556)}=8,653 \mathrm{p}=0,003\right)$. Foram identificadas interações significantes entre alguns dos fatores, tais como: lateralidade versus

\section{Condição $\mathrm{x}$ lateralidade $\mathrm{x}$ momento}
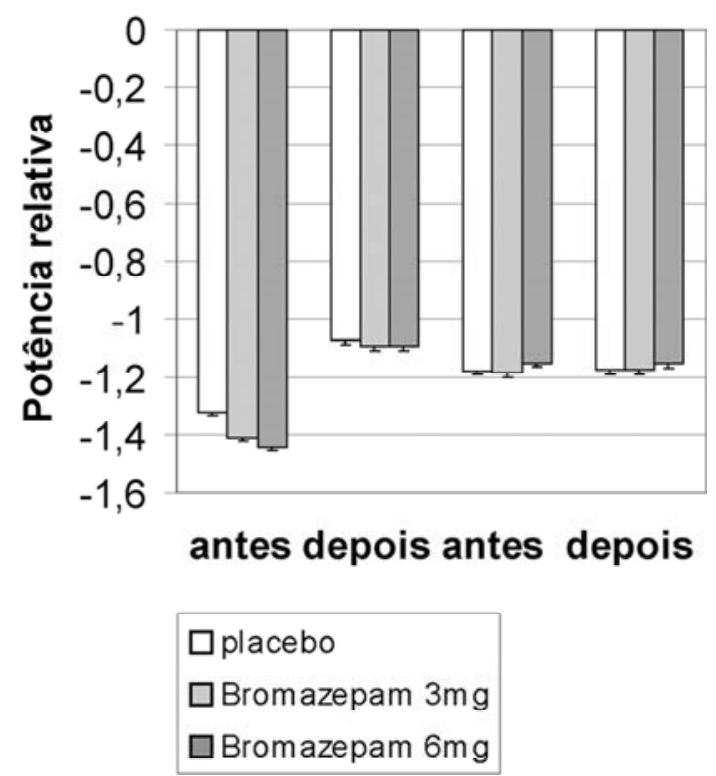

Fig 3. Relação entre condição (placebo $x$ bromazepam $3 \mathrm{mg} x$ b romazepam $6 \mathrm{mg}$ ), lateralidade (a para mão esquerda $x$ ç para a mão direita) e momento (antes $x$ depois do pressionamento da tecla de interesse).

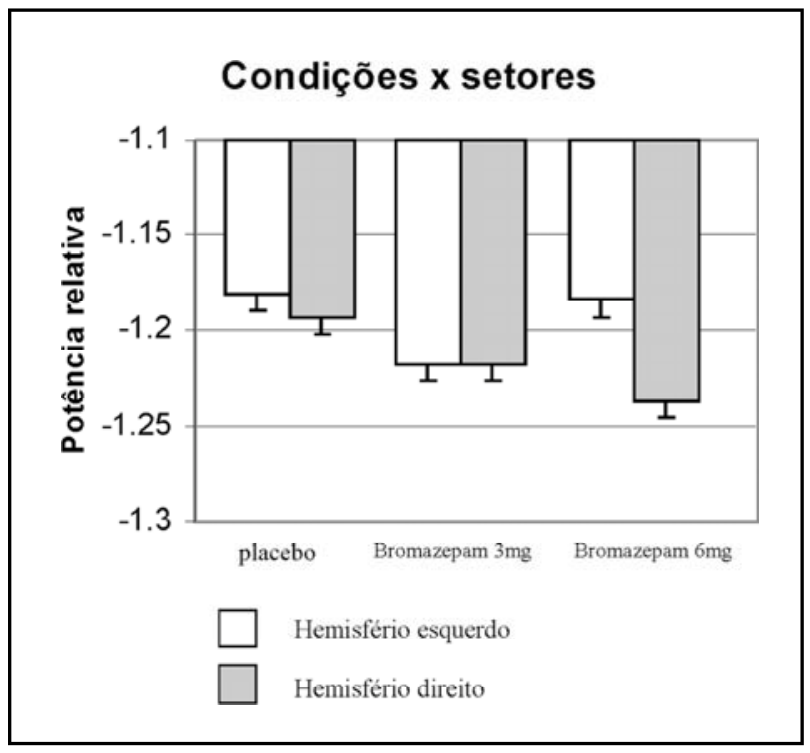

Fig 4. Relação entre as diferentes condições (placebo, bromaze pam $3 \mathrm{mg}$ e bromazepam $6 \mathrm{mg}$ ) e os setores no escalpo (hemis fério direito e esquerdo).

condição versus momento (Fig 3) $\left(F_{(3,1556)}=4,332 p\right.$ $=0,013)$; lateralidade versus condição $\left(F_{(3,1556)}=\right.$ $13,493 p=0,000)$; lateralidade versus momento $\left(\mathrm{F}_{(3,1556)}=412,975 \mathrm{p}=0,000\right)$; condição versus momento $\left(F_{(3,1556)}=3,615 p=0,027\right)$; condição versus setor (Fig 4) $\left(F_{(3,1556)}=4,754 p=0,009\right)$. Todas as outras 
combinações dentro deste modelo de ANOVA não mostraram significância, não se observando assim o u tros efeitos principais ou interações.

\section{DISCUSSÃO}

O presente estudo objetivou verificar as alterações motoras e eletrocorticais decorrentes da administração de diferentes doses de benzodiazepínico (bromazepam) mediante prática de uma tarefa motora. E ainda, relacionar o efeito da droga à performance motora da mão dominante versus mão não-dominante. O bromazepam é um benzodiazepínico agonista gabaérgico, em especial, sua ação se faz sobre os receptores do ácido gama aminobutírico ${ }^{4,7,30}$. Nesta situação o grupamento neural apresenta suas atividades parcialmente inibidas ou pelo menos dificultadas em virtude da hiperpolarização. Tal hiperpolarização pode ser intensificada com o uso do bromazepam em difere $n$ tes dosagens. Desta forma, facilita-se a ligação do neurotransmissor GABA que termina por levar a inibição neuronal e conseqüente redução dos níveis de ansiedade do sujeito ${ }^{3,8,9}$. Neste contexto, esta discussão será dividida em tópicos de acordo com os resultados obtidos.

Variável neuropsicológica - Os resultados do teste selecionado para análise da atenção sustentada (Stroop) não demonstraram diferenças entre os grupos (placebo, bromazepam 3mg e 6mg). Em particular, estas diferenças não foram verificadas em relação ao escore bruto, e também não foram verificadas em relação ao fator tempo para execução do teste. Tais resultados demonstraram que a d roga não produziu alterações nos níveis de atenção capazes de serem detectadas pelo teste. Em outros modelos o teste é uma ferramenta de valor para medidas cognitivas. Medidas psicométricas como testes de atenção e memória ${ }^{12}$, tarefas de vigilância visual ${ }^{4}$ e tempo de reação (TR) têm sido empregadas associadas ao teste de Stroop. Experimentos tem demostrado claramente que este tipo de teste atencional pode ser utilizado para se obsenar alterações em áreas pré-frontais, visto que estas relacionam-se a mecanismos modulatórios dos focos de atenção ${ }^{31}$. Memória, emoção e atenção tem sido correlacionados em outros modelos experimentais associados ao teste de Stroop. Nestes estudos nota-se claramente as relações entre os aspectos emocionais interferindo na retenção das in fo rmações como nos diversos tipos de memórias e as interferências nos níveis atencionais. Desta forma, fica evidente que a atenção é etapa funda- mental para retenção das informações a curto e a longo prazo ${ }^{32}$. Além da área pré-frontal, estudos correlacionam áreas temporais e frontais com os processos mnésicos e atencionais ${ }^{33}$.

Variáveis comportamentais - Neste estudo foram consideradas duas variáveis do tipo comport a mentais, tempo de execução da tarefa de datilografia e número de erros por bloco independente de exercício. Assim, ambas podem ser consideradas para se verificar a aquisição de procedimentos motores referentes à tarefa solicitada. $\mathrm{Na}$ análise da relação entre as condições e os blocos, não foi notada interação. A condição também não mostrou relações estatisticamente significantes. Com base nos resultados observados destaca-se, como efeito principal, o fator blocos para ambas as variáveis (tempo e erros). Este achado parece estar relacionado com o aprendizado relacionado à tarefa motora e não ao uso do bromazepam. O aspecto do efeito principal estar relacionado ao bloco aponta para alterações motoras induzidas através da aprendizagem, pois mostra alterações plásticas ocorridas em função da consolidação da informação ${ }^{21-23}$. É provável que o efeito ansiolítico da droga não tenha influenciado na performance motora, visto que na análise do fator condição (placebo versus bromazepam de $3 \mathrm{mg}$ versus bromazepam de $6 \mathrm{mg}$ ) não foram verificadas diferenças estatísticas. De maneira similar aos resultados obtidos, estudos mostram a exposição de indivíduos a testes de atenção, extensão de memória e TR utilizando bromazepam em diferentes doses (1,5mg e $3 \mathrm{mg}$ ) e placebo e não indicam prejuízo psicomotor tanto em função do medicamento quanto em função da dosagem. Ao contrário, com as doses administradas, houve leve efeito positivo na performance que pode ser explicado pela diminuição do estado de ansiedade ${ }^{10}$.

Na comparação entre os fatores lateralidade (mão esquerda versus mão direita) e os erros no terceiro bloco de exercícios, observou-se efeito principal para lateralidade. Embora todos os sujeitos tenham sido selecionados utilizando-se como critério de inclusão a dominância da mão direita, nossos resultados identificaram número maior de e rros em relação a mão dominante (direita). Os dif e rentes níveis atencionais aplicados em cada mão em tarefas motoras parecem explicar as divergências encontradas no número de erros. A princípio a suspeita (hipótese) ficava em torno das capacidades motoras da mão direita serem maiores (cometendo menos erros) do que em relação a mão 
esquerda, visto que os sujeitos eram destros. Entretanto, os erros obtidos em maior número pela mão dominante parecem evidenciar que as demandas de atenção do sujeito durante a prática foram maiores para a mão não-dominante (esque rda). As experiências comportamentais prévias dos destros em suas atividades motoras diárias talvez gerem influências que levem a um processo de condicionamento ${ }^{34}$. Por esta analogia, pode-se considerar que tarefas motoras tendem a ser realizadas com níveis atencionais mais baixos no lado dominante ${ }^{35}$.

Variáveis eletrofisiológicas - Nas variáveis eletrofisiológicas foram consideradas a banda teta e os seguintes eletrodos: F8, P4, C4, F4, Fp2, F7, P3, C3, F4, Fp1. A atividade teta foi escolhida em função da associação desta com processos de automatização dos movimentos, modalidades atencionais e aprendizagem ${ }^{18,25}$. A representação do córtex frontal e parietal foi dada pelos eletrodos eleitos para tais análises. Especificamente, eles representam respectivamente as áreas do controle motor bi-manual no que concerne a motricidade e somestesia ${ }^{36}$. Neste contexto, os resultados serão discutidos em função dos efeitos principais, e suas respectivas interações dentro do desenho experimental proposto: condição (3) (placebo x bromazepam $3 \mathrm{mg} x$ bromazepam $6 \mathrm{mg}$ ); setor (2) (hemisfério esquerdo $x$ direito); momento (2) (2 segundos antes do pressionamento das teclas $\mathrm{x} 2$ segundos depois do pressionamento das teclas); lateralidade (2) (mão direita e mão esquerda).

Interação condição versus momento versus la teralidade - As relações entre os três fatores podem ser interpretadas como interações entre o uso ou não da droga, o processamento motor cortical (antes e depois do toque no teclado) e entre as mãos (direita e esquerda). Conforme já evidenciado em experimentos anteriores a tarefa induz modificações corticais ${ }^{21-23}$. Especificamente, esta tripla interação (condição/momento/lateralidade) sugere que as condições dos processamentos motores corticais, além de serem plásticas, são diversas. O fator condição marca bem esta afirmação, já que foram significantes nos três parâmetros pesquisados (placebo/bronazapam de $3 \mathrm{mg} / 6 \mathrm{mg}$ ). E ainda, os dados demonstraram interações significativas entre a lateralidade (mão esquerda em relação à mão direita) e condição (placebo, bromazepam de $3 \mathrm{mg}$ e de $6 \mathrm{mg}$ ). Estes achados evidenciam uma relação entre a droga administrada e a tarefa manual. Particula mente, as correlações estabelecidas entre os fatores momento ( 2 segundos antes da tecla de interesse e 2 segundos depois) versus lateralidade (mão esquerda em relação à mão direita) sugere $m$ que o processamento cortical destas informações somatomotoras e somato-sensitivas ocorrem de modo distinto no que concerne ao tempo de planejamento, e a relação com a mão selecionada para execução do movimento ${ }^{35}$. De maneira mais ampla, pode-se afirmar que tarefas manuais re petidas e rápidas terminam por gerar alterações nos padrões hemisféricos quanto a lateralidade ${ }^{37}$. Tais resultados suportam a idéia de que o processamento cortical é dependente e correlaciona-se com os tempos específicos de processamento sensório-motor ${ }^{38}$. Os dados obtidos estão de acordo com outras evidências em relação aos tempos de planejamento motor no lobo frontal (áreas secundárias), e ao tempo de envio da resposta motora para realização do movimento (área motora primária) ${ }^{39}$. Estudos sugerem que as áreas de planejamento são ativadas previamente em relação às áreas motoras primárias. Num primeiro momento o córtex organiza e planeja os parâmetros relacionados aos movimentos e em seguida envia a ordem real para a realização do dado movimento ${ }^{37}$. Várias são as pesquisas que indicam a interação de regiões subcorticais no planejamento dos movimentos. Quanto à lateralidade coincidem estudos prévios com os aspectos corticais encontrados. As vias motoras corticais comandam de maneira cruzada os dois lados corporais ${ }^{37,40}$. A condição (placebo, bromazepam de 3 e $6 \mathrm{mg}$ ) e o momento do pressionamento ( 2 segundos antes e 2 depois) suportam a premissa de que a droga, por este aspecto dos dados eletrofisiológicos, altera o processamento sensório-motor para tal atividade. Ao comparar estes achados com os dados comportamentais (em que somente o bloco foi considerado como efeito principal) observa-se que a droga não afeta as programações para os resultados da tarefa em si, isoladamente (aspecto comportamental), porém o córtex, como unidade plástica que é, logo sofre adaptações face a este modelo de aprendizagem alterando seus tempos de processamento (antes e depois da respectiva tecla). Tal plasticidade cortical é fruto da enorme capacidade cortical de moldarse em função de tarefas motoras ${ }^{35}$. As análises mostraram (Post hoc), além das interações, diferenças significantes entre o grupo placebo e ambos os grupos bromazepam. Particularmente, foram evidenciadas diferenças entre os grupo placebo e o bromazepam de $3 \mathrm{mg}$ e o grupo placebo e o bromazepam de $6 \mathrm{mg}$. Não houve diferenças entre os 
grupos bromazepam de $3 \mathrm{mg}$ e de $6 \mathrm{mg}$. Estes dados indicam que a droga gerou alterações eletroc o rticais a ponto de influenciar no processamento das informações e na característica do envio motor. Fica evidente que a droga, mesmo em doses mais baixas ( $3 \mathrm{mg}$ ) gera alterações no processamento motor, e as doses mais elevadas estudadas aqui (6mg) demonstraram repercussões maiores nos parâmetros corticais.

Interação condição versus setor - Especifica mente, nota-se que a mão direita trabalha de modo diferente da mão esquerda no que diz respeito à atividade elétrica do córtex cerebral. O hemisfério esque rob nestes sujeitos foi mais ativado que o direito, visto que os valores médios de potência relativa nesta banda são inferiores ${ }^{41}$. Desta maneira identificamos sincronização de áreas à esquerda provavelmente por maior demanda de grupos neuronais a trabalharem para gerar movimentos na mão direita ${ }^{42}$. Estes achados divergem das respostas comportamentais obtidas quando comparou-se a lateralidade e o número de erros cometidos no terce iro bloco. Aspectos cognitivos podem estar influenciando nestas modulações e mesmo nos aspectos plásticos provenientes da tarefa motora ${ }^{34}$. A plasticidade é tão evidente face aos mecanismos motore, como o treinamento motor, que modifica as sinapses em áreas motoras corticais ao ponto de reter informações específicas ${ }^{35}$. Modelos celulares tem comprovado estas consolidações em áreas corticais motoras ${ }^{43}$. O bromazepam, neste modelo experimental, não alterou o córtex motor a ponto de prejudicar a tarefa motora, ao contrário, comprovou-se que sua ação, nestas dosagens, é, em parte, até benéfica. Este fato advém do caráter ansiolítico da droga, que, desta forma, parece reduzir a preocupação do indivíduo em relação ao tempo e número de erros $\mathrm{s}^{12,44,45}$. A consequência direta disto é uma melhora geral na performance do sujeito, tanto no que concerne aos aspectos comportamentais (tempo e erros da tarefa), quanto aos aspectos eletrofisiológicos (variáveis do EEG). A dominância dos sujeitos examinados pode ser considerada também como fator relevante, visto que as análises do setor (hemisfério esquerdo em relação ao direito) mostraram maior demanda de grupos neurais à esquerda ${ }^{42}$, porém as repostas motoras mais eficientes foram verificadas no hemisfério direito. Atualmente, reconhece-se a complexidade dos circuitos neurais reguladores dos mecanismos plásticos corticais voltados para questões de lateralidade ${ }^{37}$. Esta controvérsia pode advir de alguma forma do efeito do medicamento. Desta forma fica claro que a droga gerou alterações nos padrões hemisféricos no que diz respeito a lateralidade. Estudos com outras formas de mapeamento ce rebral poderiam elucidar tais mecanismos ${ }^{46}$, assim como trabalhos em nível celular ${ }^{43}$.

Em conclusão, este trabalho apoia os estudos relacionados com atividades motoras e ansiolíticos. As avaliações realizadas mostraram que o bromazepam não gera prejuízos neste modelo de tarefa. A revisão da literatura realizada mostra evidências de que a banda de frequência teta está relacionada ao aprendizado, conforme este trabalho também relata. $\mathrm{Na}$ associação realizada neste modelo notase a interação entre os dois hemisférios cerebrais para realização da tarefa, mesmo nas condições experimentais. Este desenho experimental não suporta exposição do sujeito a dois momentos distintos na tarefa; primeiro momento placebo, segundo momento experimental; devido ao efeito da aprendizagem. Outros experimentos podem ser feitos para relacionar tarefas motoras não vinculadas ao efeito direto da aprendizagem com $\mathrm{d}$ rogas ansiolíticas. Além disso, tal modelo poderia ser aplicado a um grupo de indivíduos sinistros para se estabelecer uma comparação entre os resultados obtidos. As análises estatísticas evidenciam que vários podem ser os mecanismos para se entender os fenômenos observados neste estudo. A opção feita pelos autores vem deparar-se com os objetivos do estudo, no qual pretendia correlacionar tarefa motora controlada X bromazepam X atividade cortical cerebral. De maneira geral não obsenou-se prejuízos motores e nem prejuízos atencionais. Pelo contrário os indícios demonstraram vantagens para o grupo experimental pela ótica das variáveis comportamentais. Estudos futuro $\mathrm{s}$ podem relatar os efeitos cumulativos da droga e suas repercussões nas práticas motoras.

\section{REFERÊNCIAS}

1. Brant LC, Dias EC. Work and distress among managers in a stateowned company undergoing a restructuring process. Cad Saúde Pública 2004;20:942-949.

2. McLean SA, Clauw DJ. Predicting chronic symptoms after an acute"stressor: lessons learned from 3 medical conditions. Med Hypotheses 2004;63:653-658.

3. Graeff, F. Drogas psicotrópicas e seu modo de ação, 2.Ed. São Paulo: EPU, 1989.

4. Leeuwen Van TH, Verbaten MN, Koelega HS, Kenemans JL, Slangen JL. Effects of bromazepam on single-trial event-related potentials in a visual vigilant task. Psychopharmacology 1992;106:555-564.

5. Versiani M, Nardi AE, Figueira I, Mendlowicz M, Marques C. Doubleblind placebo controlled trial with bromazepam in social phobia. J Brasil Psiquiatria 1997;46:167-171.

6. Blanco C, Antia SX, Liebowitz MR. Pharmacotherapy of social anxiety disorder. Biol Psychiatry 2002;51:109-120. 
7. Visser SJ, Post JP, Waal PP, Cornet F, Cohen AF, Gerven JM. Biomarkers for the effects of benzodiazepines in healthy volunteers. Br J Clin Pharmacol 2003;55:39-50.

8. Kandel E, Schwartz S, Jessel T. Principles of neuroscience, $4^{\text {th }}$ Ed. New York: McGraw-Hill, 2000.

9. Oelschlager H. Chemical and pharmacologic aspects of benzodiazepines. Schweiz Rundsch Med Prax 1989;78:766-772.

10. Hobi V, Dubach UC, Skreta M, Forgo I, Riggenbach H. The subacute effect of bromazepam on psychomotor activity and subjective mood. J Intern Med Res 1982;10:140-146.

11. Jansen A, Verbaten M, Slangen J. Acute effects of bromazepam on signal detection performance, digit symbol substitution test and smooth pursuit eye movements. Neuropsychobiology 1988;20:91-95.

12. Bourin M, Auget JL, Colombel MC, Larousse C. Effects of single oral doses of bromazepam, buspirone and clobazam on performance tasks and memory. Pharmacopsychiatry 1989;22:141-145.

13. Guye M, Parker GJ, Symms M, et al. Combined functional MRI and tractography to demonstrate the connectivity of the human primary motor cortex in vivo. Neuroimage 2003;19:1349-1360.

14. Baker SN, Pinches EM, Lemon RN. Synchronization in monkey motor cortex during a precision grip task: II. Effect of oscillatory activity on corticospinal output. J Neurophysiol 2003;89:1941-1953.

15. Jahnig P, Jobert M. Methodological considerations for the evaluation of EEG mapping data: a practical example based on a placebo/diazepam crossover trial. Neuropsychobiology 1995;3:31-46.

16. Oldfield R. The assessment and analysis of handedness: the Edinburgh inventory. Neuropsycology 1971;9:97-113.

17. Jasper $H$. The ten-twenty electrode system of the international federation. EEG Clin Neurophysiol 1958;10:371-375.

18. Niedermeyer E, Silva F. Electroencephalography: basic principles, clinical applications and related fields, $4^{\text {th }}$ Ed. Baltimore: Urban \& Schwarzenberg, 1999.

19. Hamzei F, Dettmers C, Rzanny R, Liepert J, Büchel C, Weiller C. Reduction of excitability ("inhibition") in the ipsilateral primary motor cortex is mirrored by fMRI signal decreases. Neuroimage 2002;17:490-496.

20. Slobounov S, Chiang H, Johnston J, Ray W. Modulated cortical control of individual fingers in experienced musicians: an EEG study. Clin Neurophysiol 2002; 113: 2013-2024.

21. Bastos VHV, Alves HVD, Piedade RAM, Silva VF, Silva APRS. Alterações corticais produzidas em função de uma tarefa de datilografia. Fit Perform 2002;1:53-58.

22. Bastos VHV, Veiga H, Cunha M, Guimarães MA, Piedade R, Ribeiro P. Assimetria inter-hemisférica em função da aprendizagem de uma tarefa de datilografia. Fisioterap Brasil 2003;4:426-431.

23. Cunha M, Bastos VHV, Veiga H, Cagy M, Piedade R, Ribeiro P. Alterações na distribuição de potência cortical em função da consolidação da memória no aprendizado de datilografia Arq. Neuropsiquiatr 2004;62:662-668.

24. Santos C. Novíssimo guia do datilógrafo. 40.Ed. São Paulo: Saraiva, 1997.

25. Caplan JB, Madsen JR, Bonhage AS, Scheibe RA, Newman EL, Kahana MJ. Human $\theta$ oscilations related to sensorimotor integration and spatial learning. J Neurosci 2003;23:4726-4736.

26. Coan JA, Allen JJ. Frontal EEG asymmetry as a moderator and mediator of emotion. Biol Psychol 2004;67:7-49.

27. Smyrnis N, Theleritis C, Evdokimidis I, Müri RM, Karandreas N. Single- pulse transcranial magnetic stimulation of parietal and prefrontal areas in a memory delay arm poiting task. J Neurophysiol 2003;89:3344-3350.

28. Schack B, Chen CAN, Mescha S, Witte H. Instantaneous EEG coherence analysis during the Stroop task; Clin Neurophysiol 1999;110:1410-1426.

29. Bucci P, Mucci A, Volpe U, Merlotti E, Galderisi S, Maj M. Executive hypercontrol in obsessive-compulsive disorder: electrophysiological and neuropsychological índices. Clin Neurophysiol 2004;115:1340-1348.

30. Zafar S, Saxena NC, Conrad KA, Hussain A. Statistical mechanics model for the interaction between the neurotransmitter gammaaminobutyric acid and GABAA receptors. Physiol Rev Lett. 2004;93:018103/1-018103/4.

31. Kato M. Prefrontal lobes and the attentional control: a neuropsychological study using modified Stroop test. Rinsho Shinkeigaku 2001;41:1134-1136.

32. MacKay DG, Shafto M, Taylor JK, Marian DE, Abrams L, Dyer JR. Relations between emotion, memory, and attention: evidence from taboo stroop, lexical decision, and immediate memory tasks. Mem Cognit 2004;32:474-488.

33. Fernandes MA, Davidson PS, Glisky EL, Moscovitch M. Contribution of frontal and temporal lobe function to memory interference from divided attention at retrieval. Neuropsychology 2004;18:514-525.

34. Hantfield BD, Haufler AJ, Hung TM, Spalding TW. Electroencephalographic studies of skilled psychomotor performance. J Clin Neurophysiol 2004;21:144-156.

35. Serrien DJ, Pogosyan AH, Brown P. Cortico-cortical coupling patterns during dual task performance. Exper Brain Res 2004;157:79-84.

36. Tecchio F, De Lucia M, Salustri C, et al. District-related frequency specificity in hand cortical representation: dynamics of regional activation and intra-regional synchronization. Brain Res 2004;1014:80-86.

37. Agnew JA, ZeffiroTA, Guinevere FE. Left hemisphere specialization for the control of voluntary movement rate. Neuroimage 2004;22:289-303.

38. Abdul-latif AA, Cosic I, Kumar DK, Polus B, Pah N, Djuwari D. EEG coherence changes between right and left motor cortical areas during voluntary muscular contraction. Australas Phys Eng Sci Med 2004;27:11-15.

39. Huang MX, Harrington DL, Paulson KM, Weisend MP, Lee RR. Temporal dynamics of ipsilateral and contralateral motor activity during voluntary finger movement. Hum Brain Mapp 2004;23:26-39.

40. Stemmer B, Vihla M, Salmelin R. Activation of the human sensorimotor cortex during erro r- related processing: a magnetoencephalography study. Neurosci Lett 2004;362:44-47.

41. Neuper C, Pfurtscheller G. Event-related dynamics of cortical rhythms: frequency-specific features and functional correlates. Intern Psychophysiol 2001;43:41-58.

42. Hlustik P, Solodkin A, Noll DC, Small SL. Cortical plasticity during threeweek motor skill learning. J Clin Neurophysiol 2004;21:180-191.

43. Luft AR, Buitrago MM, Ringer T, Dichgans J, Schulz JB. Motor skill learning depends on protein synthesis in motor cortex after training. J Neurosci 2004;24:6515-6520.

44. Rynn MA, Brawman-Mintzer O. Generalized anxiety disorder: acute and chronic treatment. CNS Spectr 2004;9:716-723.

45. Davidson JR. Use of benzodiazepines in social anxiety disorder, generalized anxiety disorder, and posttraumatic stress disorder. J Clin Psychiatry 2004;65:29-33.

46. Rogers BP, Carew JD, Meyerand ME. Hemispheric asymmetry in suplementary motor area connectivity during unilateral finger movements. Neuroimage 2004;22:855-859. 